\title{
Germanica
}

\section{La crise des relations interpersonnelles dans la littérature de langue allemande du $\mathrm{xx}^{\mathrm{e}}$ siècle}

\section{Elfie Poulain}

\section{(2) OpenEdition}

\section{Journals}

Édition électronique

URL : http://journals.openedition.org/germanica/1282

DOI : 10.4000/germanica.1282

ISSN : 2107-0784

Éditeur

Université de Lille

Édition imprimée

Date de publication : 30 juin 1998

Pagination : 9-15

ISBN : $9770984263203-22$

ISSN : 0984-2632

Référence électronique

Elfie Poulain, « La crise des relations interpersonnelles dans la littérature de lanque allemande du xx siècle », Germanica [En ligne], 22 | 1998, mis en ligne le 27 janvier 2012, consulté le 06 octobre 2020 URL : http://journals.openedition.org/germanica/1282 ; DOI : https://doi.org/10.4000/germanica.1282

Ce document a été généré automatiquement le 6 octobre 2020.

(c) Tous droits réservés 


\title{
La crise des relations interpersonnelles dans la littérature de langue allemande $\mathrm{du} \mathrm{xx}^{\mathrm{e}}$ siècle
}

\author{
Elfie Poulain
}

1 Blaise Pascal n'hésitait pas à écrire : «Le moi a deux qualités : il est injuste en soi, en ce qu'il se fait centre de tout; il est incommode aux autres, en ce qu'il veut les asservir: car chaque moi est l'ennemi et voudrait être le tyran de tous les autres. ${ }^{1}$ » À regarder les relations conflictuelles qui agitent notre monde occidental contemporain, ce constat n'a rien perdu de sa pertinence. Néanmoins, les changements intervenus au cours de l'histoire ont conduit à une transformation des structures de pensée qui orientent la vie quotidienne de nos jours et le conflit, jadis éprouvé sous la forme d'une angoisse interne, morale et religieuse, s'est doublé d'un conflit externe qui affecte l'ensemble des rapports humains et est ressenti très durement par les individus dans leurs relations interpersonnelles.

2 Si le problème des relations interpersonnelles se pose avec une acuité toute particulière à notre monde contemporain, c'est que la raison moderne, cet idéal hérité des Lumières, s'est avérée incapable d'orienter le monde et les individus comme elle pensait pouvoir le faire. La devise des Lumières : «Sapere aude! Aie le courage de te servir de ton propre entendement » est un appel, depuis longtemps intériorisé par le sujet pensant et réfléchissant qui tend à soumettre son existence à sa propre raison autonome et éclairée. Cet appel a engagé l'individu dans un processus de libération à l'égard de toute instance dogmatique, de tout rapport de domination et de servitude, de toute contrainte et de toute sujétion imposées de l'extérieur. Elle a effectivement conduit à l'émancipation théorique du sujet moderne, mais elle a fait jaillir simultanément un problème pratique : la soumission à la raison autonome ne peut pas ne pas rendre problématique l'insertion de l'individu dans la société car celui-ci ne peut plus, comme auparavant, se soumettre aux normes et aux valeurs qui régissent la communauté sans s'interroger lui-même sur la validité de ces normes et de ces valeurs. Aussi, la pensée de la raison moderne, bien qu'elle se soit centrée sur l'idée d'un 
progrès de l'humanité et de l'esprit humain, a-t-elle conduit à une mise en question généralisée de la vie individuelle et sociale. Soucieux de s'émanciper de toute tutelle et de toute autorité, de celle du couple, de la famille, des institutions, de la religion, etc. qui, dans le passé, lui offraient un refuge tout en lui dictant ses règles de conduite, l'individu contemporain cherche à s'affranchir de leurs conventions et de leurs règles de comportement, qui freinent sa liberté d'action. Il désire affirmer son autonomie et réaliser sa vie et se réaliser lui-même en ne se fiant qu'à sa propre régie et l'autre, quant à lui, tente de faire de même.

3 Aussi l'espace de prévisibilité des comportements se trouve-t-il restreint et les attentes des uns à l'égard des autres, gravement compromises. Les relations sociales, autrefois canalisées par les diverses institutions et leurs instances d'autorité, se voient, de ce fait, placées dans un horizon d'incertitude et d'indétermination. On assiste à un éclatement des valeurs : celui-ci engendre non seulement des ruptures et une dissolution des liens intersubjectifs et sociaux, mais il fait surgir également des problèmes de personnalité, autrement dit d'identité, chez les sujets eux-mêmes.

Le dilemme de l'individu contemporain se présente ainsi : il ne peut plus envisager son existence et ses rapports à autrui autrement que sous la forme d'une existence autonome, capable de s'assumer et de s'orienter sans direction étrangère à sa propre raison, mais il ne peut pas non plus ignorer les failles et les conséquences de ce processus d'émancipation. Au lieu de s'associer, comme il le souhaite, avec les autres pour former une communauté de sujets libres et solidaires, garants de la justice, il est confronté à des conflits et à des injustices que la raison moderne visait justement à éliminer, et ce, dans ses rapports interpersonnels comme dans ses rapports publics. Dépourvu de tout support externe, l'individu contemporain, affranchi des contraintes que lui imposaient les institutions et la religion, doit affronter le versant négatif de la liberté acquise. Ilse sent insécurisé et désœuvré, il succombe à la hantise de la culpabilité face au monde social qui, faute de Dieu, prend la place du juge souverain. Le résultat en est qu'il se replie sur lui-même en cherchant refuge dans l'isolement ou qu'il aboutit à des relations ambiguës, voire contradictoires où l'amour et le respect de l'autre se convertissent aisément en haine ou en indifférence totale.

La littérature, loin d'ignorer ce phénomène est, comme l'exprime Th. W. Adorno, ce en quoi «la société se cristallise $»^{2}$. Elle peut être lue comme expression sur le mode imaginaire, d'une réalité à laquelle elle est dialectiquement articulée sans être, pour autant, un reflet réel de cette dernière. Elle est une manifestation des tendances virtuelles de cette réalité qui se trouvent transposées dans des consciences individuelles, virtuelles, elles aussi. C'est à travers la poésie d'un peuple, pense M. Heidegger, que nous pouvons voir ce qu'il en est de ce peuple ${ }^{3}$. Et c'est à travers la littérature des relations interpersonnelles que nous pouvons savoir ce qu'il en est de l'homme, car l'écrivain n'est ni un moraliste, appliqué à restaurer le respect des normes antiques, ni un sociologue, préoccupé d'enregistrer par des descriptions neutres à l'égard de toutes valeurs, les manifestations objectives de l'éclatement des relations interpersonnelles. En respectant la spécificité de l'écriture, il respecte la spécificité de l'homme : il fait voir comment, malgré tout, l'être humain parvient à reconnaître dans ces relations interpersonnelles, même brisées, son seul lieu essentiel, le lieu où il a à être. Aucun des auteurs analysés dans ce volume ne faillit à cette tâche.

6 Le présent recueil rassemble des analyses consacrées aux œuvres de la littérature germanophone de ce siècle qui thématisent et refigurent dans leur monde parallèle, 
fictionnel, la crise des relations interpersonnelles en s'interrogeant sur ses causes, sur la diversité des répercussions qu'elle a sur les individus et sur la manière dont ces derniers cherchent à la maîtriser et à la surmonter.

7 Le premier groupe d'analyses renvoie à des œuvres littéraires qui constituent une véritable mise en scène romanesque de cette crise. Ces œuvres placent les personnages romanesques là où ils affrontent leurs problèmes: dans leur contexte de communication avec le monde social. L'Homme difficile de Hofmannsthal (G. Roussel) livre l'image d'une société où s'affrontent d'une part, des individus attachés aux institutions d'un monde ancien, celui des lieux clos et protecteurs de l'aristocratie viennoise, et d'autre part, les personnages d'un monde nouveau, déjà présent, ceux que l'expérience de la Première Guerre mondiale a précipités dans un vide de valeurs. C'est un monde social qui se casse et qui entraîne la dissolution des relations interpersonnelles. Les romans de HansFallada (P. Vaydat) se situent à une époque où les liens sociaux de ce nouveau monde se défont. Ils présentent l'idéal humain sous la forme de personnages intègres, dévoués au bien public qui échouent dans leur mission sociale de réparation face à l'égoïsme, à l'étroitesse d'esprit et à l'agressivité que les autres leur opposent malgré toute leur bonne volonté. Les romans de Max Frisch mettent en scène la relation instable au sein d'un couple. L'amour étant le rapport le plus élémentaire et le plus intime aux êtres, il constitue un révélateur privilégié du comportement social et des crises de l'être humain. Le roman Stiller (E. Poulain) fait voir qu'on ne peut harmoniser la relation à autrui seulement à partir de l'un des partenaires. La crise du couple y devient synonyme de crise d'identité. Dans Mein Name sei Gantenbein (A.M. Baranowski), le protagoniste recourt à une existence d'emprunt et se réfugie dans un jeu de tragi-comédie humaine où se découvre le dynamisme de l'imaginaire. La fausse cécité y est une forme de révolte qui introduit une rupture avec la vie et l'existence réelle, mais l'ironie affichée à l'égard des relations interpersonnelles s'avère impuissante à récuser à la longue le sérieux de la vie qui finit par rattraper et par briser ce couple. L'œuvre d'E. Canetti (E. Leroy du Cardonnoy) décrit l'effet de l'éclatement des relations d'amour entre des individus aseptisés, indifférents les uns aux autres. La séparation de l'intellect et du sexe y mène à la violence, à la destruction du sujet et à une fuite dans le délire. Les romans de J. Wassermann (M. Benoit) illustrent la crise des relations interpersonnelles telle qu'elle se trouve diffractée à partir de l'appartenance à une minorité rejetée, celle des Juifs. Le salut recherché dans le rapport religieux à Dieu y est déplacé dans les relations interpersonnelles, mais celles-ci s'avèrent ne pouvoir constituer qu'une forme négative, vaine et illusoire, de ce rapport, un ersatz nécessairement décevant.

8 Le deuxième groupe d'analyses manifeste l'échappée des auteurs vers l'écriture. La diffraction du sujet s'y reflète dans l'imaginaire et la réflexion individuelle des auteurs eux-mêmes ou des personnages avancés comme porte-parole de leurs propres expériences relationnelles. Si chez les autres auteurs, la solitude apparait comme le résultat inévitable de l'échec de leurs relations humaines, les Lettres à Merline (A. Camion) montrent que Rilke, au contraire, la cultive comme un bien suprême. Il vit son amour épistolaire et la mise à distance de la femme aimée comme le centre rayonnant, comme une attente et comme une écoute, qui ouvrent son être au monde. Sa création élégiaque et son attitude poétique se substituent comme dépassement et comme amour cosmique à la relation humaine véritable. La prose tardive d'I. Bachmann (H. Ott) est marquée par une crise profonde entre hommes et femmes, notamment par la relation conflictuelle entre la fille et le père " assassin ", qui conduit 
à la destruction du Moi féminin. La reprise utopique du langage y tend à restaurer la relation interpersonnelle dans une communication avec soi-même qui, pour elle, est toujours poétique. Les récits autobiographiques de Th. Bernard (c. Wulf) retracent l'expérience des crises familiales et mènent, comme chez I. Bachmann, aux confins de la mort et, finalement, à une sublimation de la vie dans l'écriture : comme réflexion chronique, celle-ci se fait monologue et interrogation radicale des crises relationnelles de sa vie personnelle. Par opposition à l'écriture interrogative, troublante de Th. Bernard, P. Handke (B. Desbrière) essaie de sécuriser la femme qui a rompu brutalement ses liens sociaux. L'imaginaire littéraire y devient le lieu de rencontre où l'écriture se substitue aux relations complexes, interpersonnelles. L'expérience visuelle, quasi filmique, des turbulences intérieures vécues met à distance de la crise vécue comme le ferait une musique répétitive et stéréotypée. La réflexion objectivante y devient un phénomène de décharge, mû par la volonté de restaurer l'unité avec soimême tout en se faisant style et genre littéraire. Les poèmes de G. Eich (S. Reb) constituent une critique radicale de la société et de la civilisation contemporaine, accusées de dévier les relations interpersonnelles, de pervertir une communication authentique et de broyer l'individu en ligotant sa liberté. Son écriture poétique s'oppose, comme antidote, au langage manipulé par le pouvoir, qui débouche sur une manipulation des esprits, voués à des certitudes sclérosantes. L'écriture ne peut être pour lui qu'une quête de vérité qui vise à restituer la liberté véritable et la dignité humaine par un dialogue authentique, poétique.

9 Tous ces auteurs font état de relations interpersonnelles brisées par une communication elle-même brisée et qui isole les individus les uns des autres. Derrière ce constat se dessine la nostalgie d'une relation idéale à l'autre. L'espoir de Hofmannsthal de voir se réaliser l'harmonie personnelle et sociale dans le couple ne semble donc pas pouvoir se confirmer dans la littérature récente, et même là où cette harmonie semble atteindre à la perfection, comme chez Rilke, force est d'avouer qu'elle ne reste qu'un ersatz qui ne saura remplacer l'harmonie réelle recherchée. Malgré tout, tous les auteurs en appellent au langage comme à une instance de médiation possible, quelle que soit la méfiance dont il peut être l'objet, en raison des volontés de puissance qui en pervertissent l'usage.

Le dialogue herméneutique avec les œuvres, tel que le développe H.G. Gadamer dans l'interview qui suit ces analyses littéraires, tend à faire du langage le lieu de rencontre et de communication authentique qui mène à une existence authentique. L'argument herméneutique est, à ses yeux, le suivant : on ne s'adonne à l'herméneutique que parce qu'on s'est aperçu que l'autre peut avoir raison. Par contre, là où il y a crise, qu'elle soit de communication ou de relations, il n'y a crise que parce qu'on pense que l'autre n'a pas raison. L'écriture littéraire de la crise renvoie, quant à elle, à une vérité interpersonnelle. On critique le fait de cette crise à partir de l'idéal d'une harmonie possible avec autrui, présumé, quant à lui, pouvoir s'identifier, lui aussi, aux attentes d'autrui dans ses actes, ses sentiments et ses pensées. L'écrivain ne critique donc la crise qu'en se réglant sur le modèle de la création poétique, d'une harmonisation de luimême, ressentie comme idéale, avec autrui. H.G. Gadamer n'hésite pas à contredire à ce sujet $\mathrm{W}$. Benjamin, en affirmant que l'aura de vérité des œuvres d'art, contrairement à la prophétie benjaminienne, est loin aujourd'hui de disparaitre : elle fascine plus que jamais. Si elle ne disparaît pas, c'est pour une unique raison : c'est parce qu'à partir d'elle, on peut encore faire aujourd'hui l'expérience de l'aura de vérité des relations interpersonnelles, même si cette expérience, confrontée aux expériences 
interpersonnelles de la vie quotidienne, ne peut demeurer qu'une expérience négative, dialectique. En identifiant ce qui est crise dans les relations interpersonnelles, les œuvres identifient le faux dans ces relations et agissent de ce fait comme critère de discernement dans le contexte de crise.

11 L'aura de vérité des relations interpersonnelles consiste à identifier ces dernières, par l'écriture comme ce qui est essentiel à l'homme : comme ce qui n'est réductible ni au noyau du sacré et de la conscience religieuse collective, ni non plus à une révélation du sujet à lui-même, à une révélation d'un être qui pourrait se réaliser comme être social, en dehors de l'espace de communication, dans des rapports à autrui que la raison lui aurait dictés d'avance. La vérité herméneutique vise ainsi à nous placer, à travers une interprétation des œuvres, en plein milieu de la vie.

12 N'était-ce pas d'ailleurs ce que désirait Kafka et ce qu'il se disait à lui-même, lorsqu'il écrivait dans son Journal :

Vivre, signifie être en plein milieu de la vie, c'est-à-dire voir la vie avec le regard par lequel je l'ai créée ${ }^{4}$ ?

\section{NOTES}

1. Blaise Pascal, Pensées, $n^{\circ} 455$.

2. Cf. Th. W. Adorno, Théorie esthétique, 1970, tr. fr. par Marc Jimenez, Paris, 1974, et « Thesen zur Kunstsoziologie », in Ohne Leitbild. Parva Aesthetica, Francfort s.M., Ed. Suhrkamp, 1967.

3. Cf. «Die Krise der Moderne:b. Martin Heidegger - durch die Dichtung ", in Geschichte der Philosophie, éd. par Gunnar Skirbekk et Nils Gilje, Francfort s.M., Ed. Suhrkamp, 1993, p. 892-896.

4. Franz Kafka, «Journal du 11 février 1918 » : «Leben heißt in der Mitte des Lebens sein ; mit dem Blick das Leben sehn, in dem ich es erschaffen habe.» 